\title{
BMJ Open Improvement of perioperative care of the elderly patient (PeriAge): protocol of a controlled interventional feasibility study
}

\author{
Cynthia Olotu, ${ }^{1}$ Lisa Lebherz, ${ }^{2}$ Martin Härter, ${ }^{2}$ Anna Mende, ${ }^{1}$ Lili Plümer, ${ }^{1}$ \\ Alwin E Goetz, ${ }^{1}$ Christian Zöllner, ${ }^{1}$ Levente Kriston, ${ }^{2}$ Rainer Kiefmann ${ }^{1}$
}

To cite: Olotu C, Lebherz L, Härter $\mathrm{M}$, et al. Improvement of perioperative care of the elderly patient (PeriAge): protocol of a controlled interventional feasibility study. BMJ Open 2019;9:e031837. doi:10.1136/ bmjopen-2019-031837

- Prepublication history and additional material for this paper are available online. To view please visit the journal (http:// dx.doi.org/10.1136/bmjopen2019-031837).

$\mathrm{CO}$ and $\mathrm{LL}$ contributed equally. LK and RK contributed equally.

$\mathrm{CO}$ and $\mathrm{LL}$ are joint first authors.

Received 21 May 2019

Revised 30 August 2019

Accepted 11 October 2019

Check for updates

(C) Author(s) (or their employer(s)) 2019. Re-use permitted under CC BY-NC. No commercial re-use. See rights and permissions. Published by BMJ.

${ }^{1}$ Department of Anaesthesiology, University Medical Center Hamburg-Eppendorf, Hamburg, Germany

${ }^{2}$ Department of Medical

Psychology, University Medical Center Hamburg-Eppendorf, Hamburg, Germany

Correspondence to

Lisa Lebherz; I.lebherz@uke.de

\section{ABSTRACT}

Introduction Geriatric patients have a pronounced risk to suffer from postoperative complications. While effective risk-specific perioperative measures have been studied in controlled experimental settings, they are rarely found in routine healthcare. This study aims (1) to implement a multicomponent preoperative and intraoperative intervention, and investigate its feasibility, and (2) exploratorily assess the effectiveness of the intervention in routine healthcare.

Methods and analysis Feasibility and exploratory effectiveness of the intervention will be investigated in a monocentric, prospective, non-randomised, controlled trial. The intervention includes systematic information for patients and family about measures to prevent postoperative complications; preoperative screening for frailty, malnutrition, strength and mobility with nutrient supplementation and physical exercise (prehabilitation) as needed. Further components focus on potentially inadequate medication, patient blood-management and carbohydrate loading prior to surgery, retainment of orientation aids in the operating room and a geriatric anaesthesia concept. Data will successively be collected from control, implementation and intervention groups. Patients aged $65+$ with impending surgery will be included. A sample size of $240, n=80$ per group, is planned. Assessments will take place at inclusion and 2, 30 and 180 days after surgery. Mixed-methods analyses will be performed. Exploratory effectiveness will be assessed using mixed segmented regressions. The primary endpoint is functional status. Secondary endpoints include cognitive performance, health-related quality of life, length of inpatient stay and occurrence of postoperative complications. Feasibility will be assessed through semi-structured interviews with staff and patients and quantitative analyses of the data quality, focussing on practicability, acceptance, adoption and fidelity to protocol. Ethics and dissemination The study will be carried out in accordance with the Helsinki Declaration and to principles of good scientific practice. The Ethics Committee of the Medical Association Hamburg, Germany, approved the protocol (study ID: PV5596). Results will be disseminated in scientific journals and healthcare conferences.

Trial registration number ClinicalTrials.gov Identifier: NCT03325413.
Strengths and limitations of this study

- Feasibility and exploratory effectiveness evaluation of a multicomponent preoperative and intraoperative intervention under real-life circumstances for a variety of surgeries and with few inclusion restrictions.

- High patient relevance due to the use of a wide range of patient-reported outcome measures and long-term follow-up.

- Capturing multidisciplinary experience from anaesthetists, medical assistants, nurses and patients.

- Difficulties to implement and control for all intervention components adequately due to real-life circumstances.

- Risk of selection and attrition bias due to the nonrandomised design and selective dropout.

\section{INTRODUCTION}

In Germany, every second inpatient surgical procedure is performed on patients aged 65 years and above. ${ }^{1}$ This cohort has an elevated risk to suffer from a range of postoperative complications (POCs). ${ }^{2-6}$ These include postoperative delirium (POD), pulmonary infection, cardiovascular events and an overall higher rate of postoperative morbidity, consequentially extended hospitalisations, and mortality, but also long-term general decline of health, cognition, functional status and quality of life after surgery. ${ }^{7-11}$ Further, immediate POCs can result in and amplify longterm decline of health and long-term loss of functional independence and quality of life. The most common patient-related risk factors are a reduced functional status, (ie, sensory and cognitive impairment, poor physical fitness and mobility, malnutrition, polypharmacy and multimorbidity). ${ }^{12-15}$ Treatmentassociated risk factors include excessive fasting prior to surgery, dehydration, disorientation, disturbed sleep-wake-cycle, inadequate medication, anxiety, mental overload 
and stress, pain, hypothermia, loss of sensory orientation during inpatient stay ${ }^{16}$ and high invasiveness of the anaesthetic procedures and surgery.

In order to reduce POCs and generally improve clinical outcomes in elderly patients, it is important to detect patient-related risk factors prior to surgery and implement appropriate prophylactic measures. Accordingly, risk-specific prehabilitative interventions need to find their way into routine healthcare. ${ }^{12}$ Evidence is consistent that preoperative prehabilitative measures can reduce the postoperative risk suffering POCs for elderly patients and hence improve long-term functional status. Protective measures include countering malnutrition, ${ }^{17} 18$ poor physical fitness ${ }^{19} 20$ and enhancing breathing exercise techniques, ${ }^{21}$ as well as reducing potentially inappropriate or multi-medication. ${ }^{22}{ }^{23}$ Handling of preoperative fasting is another problematic aspect of perioperative care. While guidelines support that 6 hours of preoperative fasting are sufficient in most cases, this is hardly met in clinical practice. ${ }^{24} 25$ Recent studies, however, point out the protective effect of preoperative carbohydrate intake on the postoperative outcome, especially in vulnerable patients. ${ }^{26}$ Further risk factors for less favourable postoperative outcomes are anxiety and psychological and mental stress. While the necessity of an inpatient surgery alone provokes a stress reaction, so does the entire medical procedure, from preanaesthetic evaluation to inpatient discharge, bolstered by the unfamiliar environment and the uncertainty of the outcome. This holds particularly true for potentially vulnerable patient groups, as is the geriatric cohort. Stress is well established to negatively impact somatic and mental health outcomes. ${ }^{27}$ However, loss of orientation and high levels of stress can be reduced by marginal changes in routine preoperative procedures. Patients can be reoriented by retaining glasses and hearing aids up to the anaesthetic induction, and by reducing mental stress and overload. This can be done by ensuring that the patient understands the procedures for surgery and therapy and by encouraging the presence and involvement of relatives, ${ }^{28}$ which in turn may lead to a higher preservation of preoperative self-reliance and health-related quality of life. ${ }^{29}$

Further, the risk of different intraoperative procedures should be taken into consideration. The risk of suffering POCs is increased in patients, who have blood deficiency states and undergo sanguineous surgery, this risk can be reduced by individualised iron substitution. ${ }^{30-33}$ It is recommended to monitor the depth of anaesthesia using for example, bispectral index (BIS) analysis, as deep anaesthesia is associated with a higher incidence of postoperative delirium. ${ }^{34}$ Postoperative pain is a predisposing factor for POCs. ${ }^{35}$ To enable sufficient postoperative, opioid-saving analgesia, the use of catheter-assisted regional anaesthesia is preferable for elderly patients. ${ }^{32} 36$

While these risk factors are well studied and several intervention components have been shown to reduce complication rates in controlled research settings, ${ }^{37-39}$ many effective intervention components are not used in routine care, ${ }^{4041}$ as both an extensive preoperative risk assessment and the administration of preoperative and intraoperative measures are time-consuming and costly.

To improve the geriatric patient's postoperative safety and health, the preanaesthetic evaluation needs to be updated to the current state of research of risk and preventive factors. Feasibility and benefit of an extended preanaesthetic evaluation and the ensuing administration of corresponding prophylactic interventions need to be demonstrated, in that it is possible to improve the preoperative and intraoperative care of geriatric patients with feasible effort, leading to an overall reduction in long-term physical and cognitive complications as well as a reduced hospitalisation period.

\section{Objectives}

In this study, a demand-based and risk-based intervention (PeriAge intervention) is developed and implemented into routine healthcare.

Objective (1) is to assess and provide exploratory evidence of the effectiveness of the PeriAge intervention, improving the postoperative outcome of a sample of elderly patients at a university hospital in Germany. The primary outcome is the change in the autonomous functioning after surgery, measured via the Instrumental Activities of Daily Living (IADL, Lawton and Brody, 1969). ${ }^{42}$ The corresponding primary hypothesis is that individualised care of the patient as part of the PeriAge intervention enhances postoperative autonomy in comparison to the control group. We expect a smaller reduction of the IADL score in the experimental condition after 1 and 6 months. Additionally, we will test the composite effect of the PeriAge intervention on POCs, cognitive performance, length of inpatient stay and several patientrelevant outcomes elaborated below.

Objective (2) of our study is to investigate the feasibility ${ }^{43}$ of the PeriAge intervention, specifically its implementation and realisation in ongoing hospital operations. We intend to show that it is possible to implement a multidimensional intervention into routine care and identify main challenges of implementation. The feasibility of the implementation is categorised after the elements practicability, acceptance, adoption and fidelity to protocol.

\section{METHODS AND ANALYSIS \\ Study design}

The PeriAge intervention will be evaluated in a monocentric, non-randomised, controlled study. The study consists of three successive arms, each 6 months in lengths (see figure 1), while lengths of arms remain subject to extension as required. Patients will be allocated in a predefined order; the project starts with the usual routine healthcare as control, followed by the implementation phase and concluded by the intervention phase. Simultaneous to the control phase, the individual components of the PeriAge intervention will be elaborated, and their implementation prepared. The 


\section{IMPLEMENTATION}

STATUS

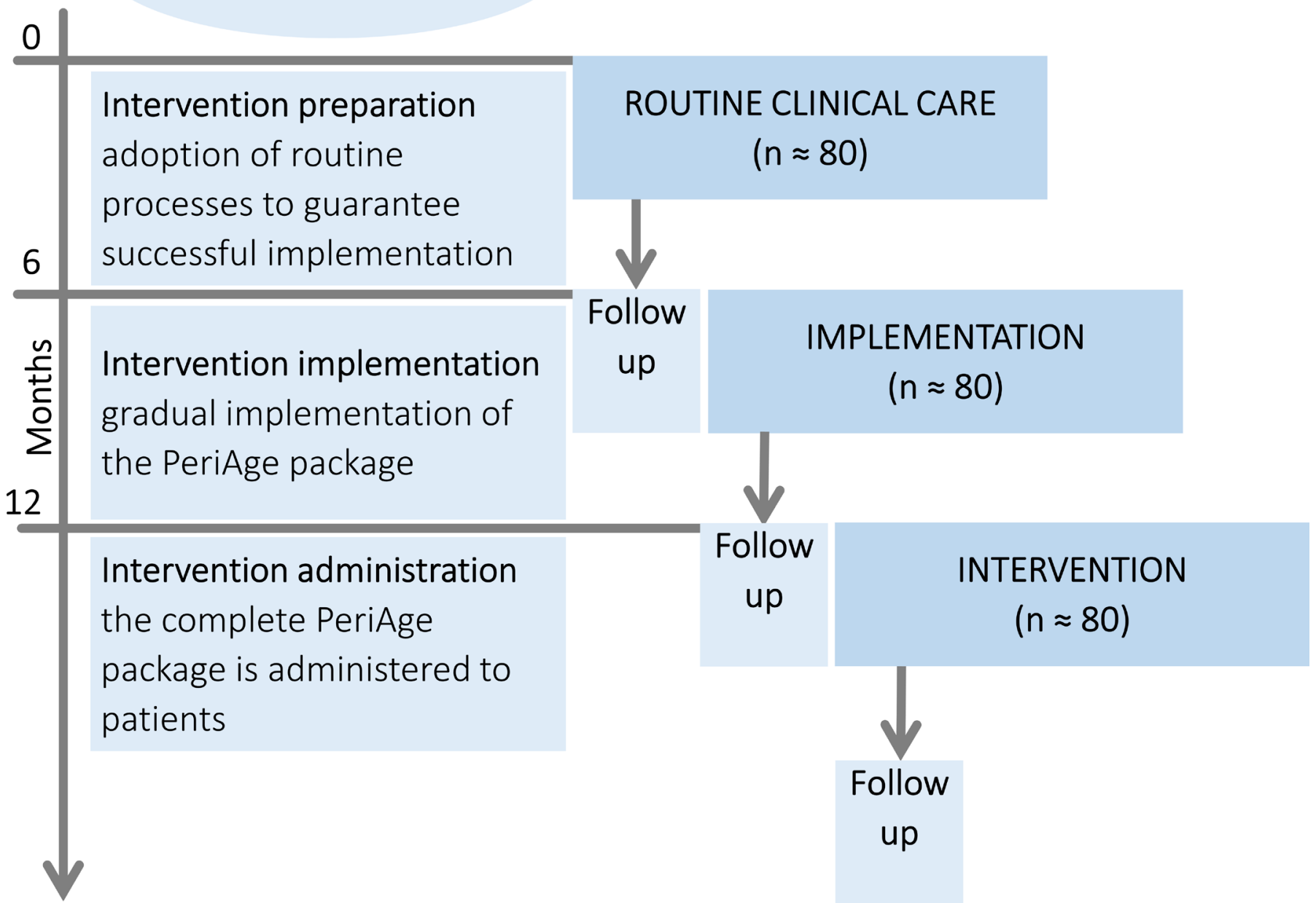

Figure 1 Sequential study design. Allocation randomisation is not feasible, due to the risk of contamination or crossover between groups. During the control and implementation phase, the intervention components will be developed, the implementation planned and gradually introduced. In the intervention phase, the exhaustive intervention will be applied. The enquiry period, entailing recruitment and follow-up of all phases, will be realised within 18 months.

implementation phase is used to implement the PeriAge intervention into routine care gradually, leaving space for adoption, tailoring and modifications as necessary. With the start of the intervention phase onwards, the final PeriAge intervention will be administered and information of its feasibility will be gathered. The 3 year mixed-method project comprises two simultaneous branches, evaluating the feasibility and effectiveness of the PeriAge intervention, respectively. For reasons of clarity and comprehensibility, the exploratory effectiveness evaluation will be discussed first.

\section{Study population}

Participants are patients aged above 64 with impending elective surgery in a university hospital of a German metropolitan region. In order to test the PeriAge intervention with high external validity, patients receiving all types of surgeries except for neurocerebral and ophthalmological surgeries will be included. While cognitive performance and functional status cannot be independently attributable to the interventions after neurocerebral surgeries, ophthalmological surgeries take place at an external site within the university medical centre and execution of intraoperative interventions cannot be guaranteed. Exclusion criteria are emergency surgery, surgery within 5 days of study inclusion (premedication visit), and surgery with planned postoperative intensive care unit admission or planned postoperative hospitalisation for fewer than 24 hours. Patients that undergo the enhanced recovery after surgery ERAS programme $^{44}$ are excluded. Further, patients will be excluded who are analphabetic, who do not have sufficient command of the German language and patients who suffer from psychosis, illicit drug use, chronic use of benzodiazepines and patients who suffer from an incorrigible auditory or visual disability. 
Effectiveness assessment of the PeriAge intervention and its influences

Procedures and instruments

Within each arm, the study follows a pre-post design. Patient assessments take place once before intervention initiation and at three time points after intervention completion as shown in figure 1 . All patients will undergo an extensive preanaesthetic evaluation (T0). In addition to the routine check-up, the assessment entails brief neuropsychological testing, to evaluate the patient's cognitive state, strength and mobility testing and patientreported outcome measures (PROMs) about somatic and mental health, current living situation and quality of life (see table 1). Additionally, the responsible anaesthetist will record malnutrition, demographics and the need for sensory aids. In the implementation and intervention group the PeriAge intervention will be introduced. However, the implementation group is merely recruited

Table 1 Multidimensional perioperative assessment; instruments, type and time point of enquiry and direction of hypothesised effect

\begin{tabular}{|c|c|c|c|c|c|c|c|}
\hline \multirow[b]{2}{*}{ Domain } & \multirow[b]{2}{*}{ Instrument } & \multirow[b]{2}{*}{ Operationalisation } & \multicolumn{4}{|c|}{ Time point } & \multirow{2}{*}{$\begin{array}{l}\text { Expected } \\
\text { direction of } \\
\text { effect }^{*}\end{array}$} \\
\hline & & & TO & T1 & T2 & T3 & \\
\hline \multirow{7}{*}{$\begin{array}{l}\text { Social, physical } \\
\text { and autonomous } \\
\text { functioning }\end{array}$} & $\mathrm{IADL}^{42} \dagger$ & Functional status & $x$ & & $x$ & $x$ & $\uparrow$ \\
\hline & $\begin{array}{l}\text { Social situation by } \\
\text { Nikolaus }^{76}\end{array}$ & Social status & $x$ & & & & N/A \\
\hline & $1 \mathrm{~min}$ sit to stand test ${ }^{4951}$ & Mobility & $x$ & & $x$ & $x$ & $\uparrow$ \\
\hline & Timed up \& go test ${ }^{77}$ & Physical strength, stamina & $x$ & & $x$ & $x$ & $\uparrow$ \\
\hline & $\begin{array}{l}\text { Vigorometer (hand } \\
\text { force) })^{50}\end{array}$ & Physical strength & $x$ & $x$ & $\mathrm{x}$ & $x$ & $\uparrow$ \\
\hline & LUCAS-Fl' ${ }^{78}$ & Frailty proxy & $x$ & & $x$ & $x$ & $\downarrow$ \\
\hline & MNA-SF ${ }^{79}$ & Malnourishment & $x$ & & & & $\mathrm{~N} / \mathrm{A}$ \\
\hline \multirow{5}{*}{$\begin{array}{l}\text { Orientation } \\
\& \text { cognition }\end{array}$} & CAM-ICU ${ }^{80}$ & Delirium & & $x$ & & & $\downarrow$ \\
\hline & DemTect ${ }^{45}$ & Cognitive functioning & $x$ & $x$ & $x$ & $x$ & $\uparrow$ \\
\hline & TAP alertness subtest ${ }^{46}$ & & $x$ & $x$ & $x$ & $x$ & $\uparrow$ \\
\hline & $\mathrm{TMT}^{47}$ & & $x$ & $x$ & $x$ & $x$ & $\uparrow$ \\
\hline & $\begin{array}{l}\text { Subjective cognitive } \\
\text { rating }\end{array}$ & $\begin{array}{l}\text { Sense of cognitive } \\
\text { functioning }\end{array}$ & $x$ & $x$ & $x$ & $x$ & $\uparrow$ \\
\hline \multirow[t]{3}{*}{$\begin{array}{l}\text { Quality of life } \\
\& \text { mental health }\end{array}$} & SF-12 4881 & $\begin{array}{l}\text { Health-related quality of } \\
\text { life }\end{array}$ & $x$ & & $\mathrm{x}$ & $x$ & $\uparrow$ \\
\hline & $\mathrm{GDS}^{82}$ & Depressive symptoms & $x$ & & $x$ & $x$ & $\downarrow$ \\
\hline & GAD- $2^{83}$ & Anxiety symptoms & $x$ & & $x$ & $x$ & $\downarrow$ \\
\hline \multirow[t]{6}{*}{ Somatic POCs } & POSPOM $^{84}$ & $\begin{array}{l}\text { Postoperative mortality } \\
\text { risk scoring }\end{array}$ & $\mathrm{x}$ & & & & $\mathrm{N} / \mathrm{A}$ \\
\hline & $\begin{array}{l}\text { Patient blood } \\
\text { management } \ddagger\end{array}$ & $\begin{array}{l}\text { Deficiency states ( } \mathrm{Hb} \text {, } \\
\text { transferrin, ferritin) }\end{array}$ & $x$ & & & & N/A \\
\hline & EPR $\ddagger$ & $\begin{array}{l}\text { Somatic complications } \\
\text { (including mortality) }\end{array}$ & & $\mathrm{x}$ & $\mathrm{x}$ & $x$ & $\downarrow$ \\
\hline & EPR & Length of hospitalisation & & $\mathrm{x}$ & & & $\downarrow$ \\
\hline & history assessment & Polypharmacy & $x$ & & & & $\mathrm{~N} / \mathrm{A}$ \\
\hline & IADL $†$ & Functional status & $\mathrm{x}$ & & $\mathrm{x}$ & $\mathrm{x}$ & $\uparrow$ \\
\hline
\end{tabular}

${ }^{*}$ The expected effect refers to the comparison between control and intervention group. An up-pointing arrow connotes a reduced respective decline in the intervention group, it does not stand for more favourable values after surgery per se.

†Primary effectiveness outcome, all instruments that are administered at T3 and the CAM-ICU will be interpreted as secondary outcomes $\neq$ Does not fit the description of an instrument, but is listed here for completeness.

CAM-ICU, Confusion Assessment Method for Intensive Care Units (Ely, Margolin, Francis et al, 2001); DemTect, Dementia Detection (Kalbe, Kessler, Calabrese et al, 2004); EPR, electronic patient record; GAD-2, generalized anxiety disorder 2 (Spitzer, Kroenke, Williams et al, 2006); GDS, Geriatric Depression Scale (Yesavage, Brink, Rose et al, 1982); IADL, Instrumental Activities of Daily Living; LUCAS-I, Longitudinal Urban Cohort Age Study - Instrument (Dapp, Anders, von Renteln-Kruse et al, 2012); MNA-SF, Mini Nutritional AssessmentShort From(@Nestlé Nutrition Institute, 1993); POC, post-operative complications; POSPOM, Preoperative Score to Predict Postoperative Mortality (Le Manach, Collins, Rodseth et al, 2016); SF-12, Short Form (12) health survey (Bullinger and Kirchberger, 1998); TAP, Test battery for attentional performance (Zimmermann and Fimm, 1993); TMT, Trail Making Test (Reitan and Wolfson, 1992). 
to gradually introduce and adjust the intervention if necessary, to guarantee a fully working and unbiassed intervention during the assessment period of the intervention group.

The first postoperative enquiry takes place (T1) within the first few days after surgery. At that point, delirium, ${ }^{45}$ cognitive functioning, ${ }^{46-48}$ physical strength $^{49} 50$ and mobility ${ }^{51}$ are assessed and information about somatic complications is extracted from the hospital's electronic patient record. POD is screened for using the Confusion Assessment Method for the Intensive Care Unit including modified Richmond Agitation and Sedation Scale in the first 5 days following surgery according to guideline recommendations. ${ }^{52} \mathrm{~T} 2$ and T3 take place 1 and 6 months after surgery, respectively.

Short-term outcomes are duration of inpatient stay, and the occurrence of postoperative complications, including POD and mortality. PROMs and a brief neurocognitive assessment, evaluating patient's postoperative cognitive abilities will be used as parameters to assessing long-term effects of the intervention, 1 and 6 months after surgery. PROMs are used to assess functional status, a proxy for frailty, health-related quality of life and mental morbidity; the neurocognitive assessment focusses on alertness, cognitive flexibility and working memory. See table 1 for instruments, operationalisation, time point of assessment and expected direction of effects.

The proposed intervention components affect either the preoperative or the intraoperative phase. While all intervention components shall counteract POC and decline of autonomy 1 and 6 months after surgery, the specific measures focus on different aspects of postoperative health. Special attention is given to everyday functioning; including nutritional and fitness status, orientation and somatic complications.

Malnourished patients will be provided with highprotein drinks for a maximum of 14 days up to the eve of their surgery day. Additionally, patients are offered a carbohydrate drink on the eve and 2 hours prior to surgery, ${ }^{53}$ but also to reduce preoperative anxiety and discomfort. ${ }^{53} 54$ Patients with frailty and poor physical fitness are prompted to undergo preoperative progressive strength and fitness training, instructed via a short personal introduction and information brochures and logged by a self-report diary. All patients are advised to perform breathing exercises, as taught by an information brochure.

\section{Interventions}

Intervention components to reduce mental overload and prevent disorientation comprise the inclusion of relatives, extensive information giving about planned procedures and the preservation of sensory orientation. The systematic inclusion of relatives or significant others in all procedures from the beginning of the inpatient stay onwards shall counteract potential disorientation within the unfamiliar, and potentially highly stressful setting. A detailed and comprehensible preoperation counselling including information about the inpatient stay and the scheduled POC prevention measures shall serve as an additional orientation aid. Patients will be encouraged to bring personal items at admission, such as pillows, photographs and music. This shall support recognition and diminish the risk of suffering POD. Furthermore, patients with need for vision aids, acoustic instruments and dental prostheses are encouraged to retain these aids up to the anaesthetic induction to foster sensory orientation.

Measures to prevent somatic complications consist of screening and potential adjustment of potentially inadequate or multi-medication in accordance with national and international recommendations ${ }^{22} 23$ and general refrainment from administering benzodiazepines. Patients with anaemia will be screened for iron deficiency. If an iron deficiency anaemia is diagnosed and the risk for intraoperative bleeding is estimated to be above $10 \%$, patients will be supplemented with intravenous iron prior to surgery in accordance of the principles of Patient Blood Management.

The proposed intraoperative measures shall prevent somatic complications and mental disorientation. The geriatric anaesthesia concept includes employing regional anaesthesia alone or in combination with general anaesthesia whenever possible to ensure an opioid-saving postoperative analgesia regime. When general anaesthesia is performed, BIS is used for neuromonitoring purposes. Further, certain medications will be avoided intraoperatively, in particular, benzodiazepines, atropine, anticholinergics and central alpha-agonists. If muscle-relaxants are needed, short-acting substances are preferred as well as postoperative catheter-assisted analgesia. Thermal blankets from anaesthesia induction to post anaesthesia care will be given to the patient in order to avoid hypothermia.

During the implementation and intervention phases, training events by study staff and external experts will be performed at every affected hospital ward and in anaesthesia meetings. These meetings inform about relevant topics of inpatient care such as the preoperative administration of carbohydrate drinks, measures of POD prevention, patient information and adequate postoperative analgesia in the elderly. Anaesthetists are instructed to follow the comprehensive administration of BIS during surgery.

\section{Recruitment/sample size}

In this trial the sample size is motivated by having a reasonable amount of patients undergoing the intervention in order to descriptively and qualitatively describe if the intervention is feasible for being executed in the routine healthcare. Nevertheless with this sample size we will reach sufficient power for explanatorily identifying rare foreseen and unforeseen incidents, as suggested for feasibility trials. ${ }^{556}$ The emergence of POCs depends on underlying conditions and type of surgery conducted. In the elected cohort, the likelihood of an occurrence of POCs is considerably above $10 \%,{ }^{5758}$ so is the risk of losing the level of preoperative functioning and autonomy. A 
sample size of 30 is minimally required for the identification of an event with an average occurrence of $10 \%$ with a confidence of $95 \% .{ }^{55}$ Because of an expected dropout greater than $30 \%$, as is common in studies that are performed under routine conditions, together with the plan to analyse multiple outcomes, we aim to recruit 80 patients in each of the three study arms, resulting in approximately 240 patients in total. The effect size of our intervention in our sample is not known as in its present combination it has not yet been tested. However, sufficiently powered effectiveness studies investigating similar populations to ours, aspects of our intervention and/or on parts of the here assessed complications, came up with similar sample sizes. ${ }^{59} 60$

\section{Data analysis}

For the exploratory effectiveness of the intervention, a comparison between the control and the intervention group will be conducted. We plan to use the intention to treat method to conduct the primary analyses. Missing values will be accounted for by using mixed modelling techniques. The data will be analysed using descriptive and inferential statistics. The effects of the intervention will be estimated by using segmented regressions. ${ }^{61-63}$ For the effectiveness analyses, generalised two-level regression models (linear, logistic or Cox depending on the outcome) will be used. This enables a nuanced estimation of time and intervention effects, taking into account time trends within and between the groups. The first level connotes the progression of the individual patients and will be estimated in intercept and slope. The second level connotes the difference between persons, taking into account time and group-effects. Should the assumptions for segmented regressions be violated, the models will be adjusted accordingly. Propensity score methods will be used in case of strong violation. ${ }^{64}$ Results with $\mathrm{p}<0.05$ will be considered as statistically significant. As this study is of explorative nature, no adjustments will take place for multiple testing. However, the elevated risk of an occurrence of type-I errors will be regarded when interpreting the results.

\section{Feasibility assessment of the implementation Procedures and instruments}

A process evaluation is conducted to explore the feasibility of the PeriAge intervention. The critical elements for capturing the degree of feasibility in this study are acceptance of those affected, in particular patients and clinical staff, as well as the, practicability, realisation and adoption, accessibility of the intervention and fidelity to protocol, chosen by means of the current standards of feasibility studies (see table 2). ${ }^{65-67}$

Using a mixed method approach, the feasibility evaluation is segmented into a quantitative and a qualitative analysis. The quantitative analysis consists of continuous documentation of the realisation of the intervention from the implementation phase onwards (see figure 2).

An intervention checklist is filled in for every patient. This checklist is tailored on risk factors and interventions of the study and enquires about the proper execution of

Table 2 Quantitative and qualitative feasibility assessment; type and description of analysis

\begin{tabular}{|c|c|c|c|c|}
\hline \multirow[b]{2}{*}{ Domain } & \multirow[b]{2}{*}{ Operationalisation } & \multirow{2}{*}{$\begin{array}{l}\text { Quantitative analysis } \\
\text { Brief description }\end{array}$} & \multicolumn{2}{|c|}{ Qualitative analysis* } \\
\hline & & & Staff & Patient \\
\hline Acceptance & $\begin{array}{l}\text { Satisfaction with the intervention and } \\
\text { its implementation }\end{array}$ & -- & $x$ & $x$ \\
\hline Practicability & $\begin{array}{l}\text { Relevance of the intervention and } \\
\text { compatibility with the specific setting }\end{array}$ & (Effectiveness outcomes, see above) & $x$ & $x$ \\
\hline $\begin{array}{l}\text { Realisation and } \\
\text { adoption }\end{array}$ & $\begin{array}{l}\text { Realisation: intend and action to } \\
\text { employ the intervention } \\
\text { Adoption: adjusted execution of the } \\
\text { intervention to fit the setting and } \\
\text { recording of these adjustments }\end{array}$ & $\begin{array}{l}\text { Data quality analysis on } \\
\text { congruency, completeness, } \\
\text { plausibility and sources of potential } \\
\text { errors. } \\
\rightarrow \text { reported and adapted if } \\
\text { necessary } \\
\text { Descriptive statistics of self- } \\
\text { report diary and intervention } \\
\text { checklist }\end{array}$ & $x$ & \\
\hline Fidelity to protocol & $\begin{array}{l}\text { Quality and of intervention delivery and } \\
\text { adherence to implementation protocol }\end{array}$ & $\begin{array}{l}\text { Evaluation of implementation } \\
\text { processes and interim adaptations by } \\
\text { intervention checklist records }\end{array}$ & $x$ & \\
\hline
\end{tabular}

*Thematic analysis of semi-structured interviews. 
IMPLEMENTATION

STATUS
FEASIBILITY

ASSESSMENT
0

Intervention preparation

adoption of routine

processes to guarantee

6 successful implementation

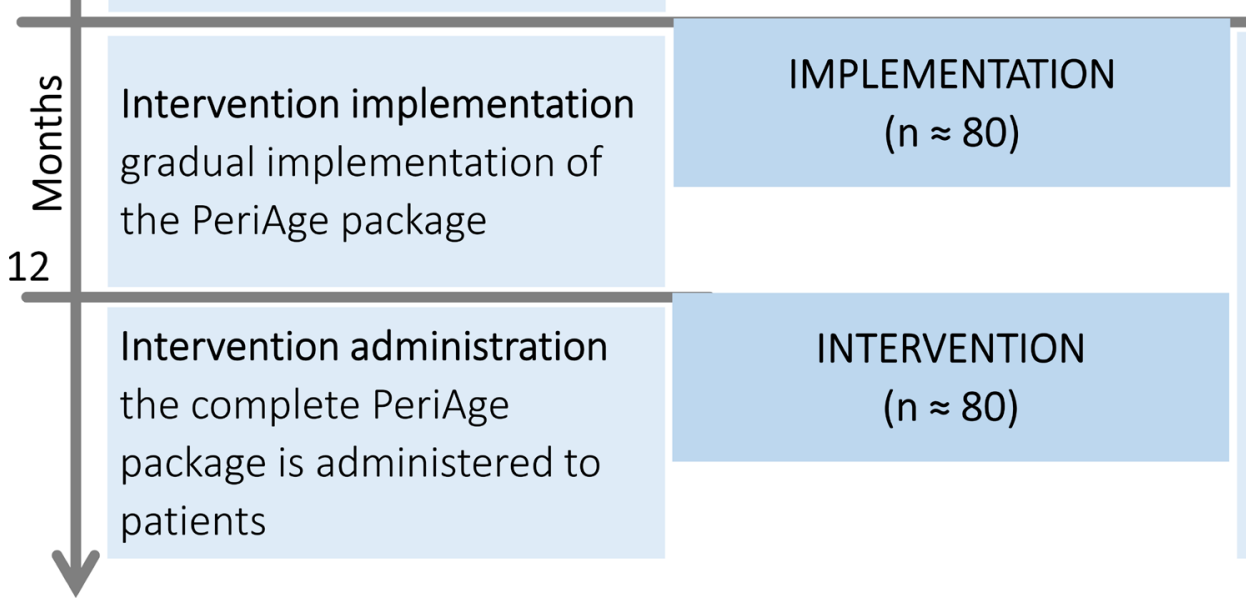

ROUTINE CLINICAL CARE $(n \approx 80)$
Quantitative: checklist \& plausibility checks Qualitative: interviews with patients and staff Outcomes: Feasibility fidelity acceptance practicability realisation accessibility

Figure 2 Incorporation of the implementation and feasibility assessment within the study outline. From the implementation phase onwards up to the completion of the intervention phase, the quantitative and qualitative feasibility analyses will be performed.

individual interventions for example, the reduction of inappropriate polypharmacy, the retainment of orientation aids and the usage of the BIS during surgery. With this collection of process data deviations from the protocol can be prevented, or alternatively, detected. Additional plausibility analyses of the outcome data are performed.

For the qualitative feasibility analyses, information on the experience of the clinical and study staff and patients regarding the individual intervention components are collected and evaluated. First, meeting logs of the project will be described. Second, semi-structured interviews will be conducted examining experience and opinion of the interviewee about adequacy and purpose of the intervention, as well as impediments and facilitators of the implementation process. The interviews will contain mainly open-ended questions. Interviewing patients and professionals of different contexts shall capture different perspectives on the implementation and increase the validity of the results. While the patient interviews will be held within the intervention phase after completing the T3 enquiry, the staff interviews will be conducted twice; once during the implementation phase and once after the termination of the intervention phase. The first staff interview serves not only as an inspection of feasibility, but also allows that necessary adjustments might be exposed and realised. The second interview repeats and finalises the inspection of feasibility.

\section{Recruitment/sample size}

Additional to the recruitment of 240 patients for the effectiveness analysis, it is planned to interview 5 to 10 study staff members medical assistants and clinicians, who are affected by the implementation. Additionally, seven randomly chosen patients of the intervention phase will be interviewed. These interviews take place after T3. The chosen sample size is based on experience and literature on saturation of information gain. ${ }^{68}$

\section{Data analysis}

To perform the process evaluation, two structured analyses of the process and outcome data will be performed on congruency and completeness in order to detect potential discrepancies between conception and realisations. The first analysis is conducted before initiation of the implementation phase and the second is conducted after the data collection is completed. The results of the evaluations as well as the results of the intervention checklist (see above), will be examined via descriptive statistics. The interviews will be recorded, transcribed and analysed by using a realist thematic analysis approach, ${ }^{69}$ specifically a framework content analysis. ${ }^{70}$ The thematic analysis approach is a method by which qualitative data is coded into themes (see figure 3 ). We will use a mainly deductive approach, as our feasibility outcomes are already predefined (see table 2). Coding schemes are developed 


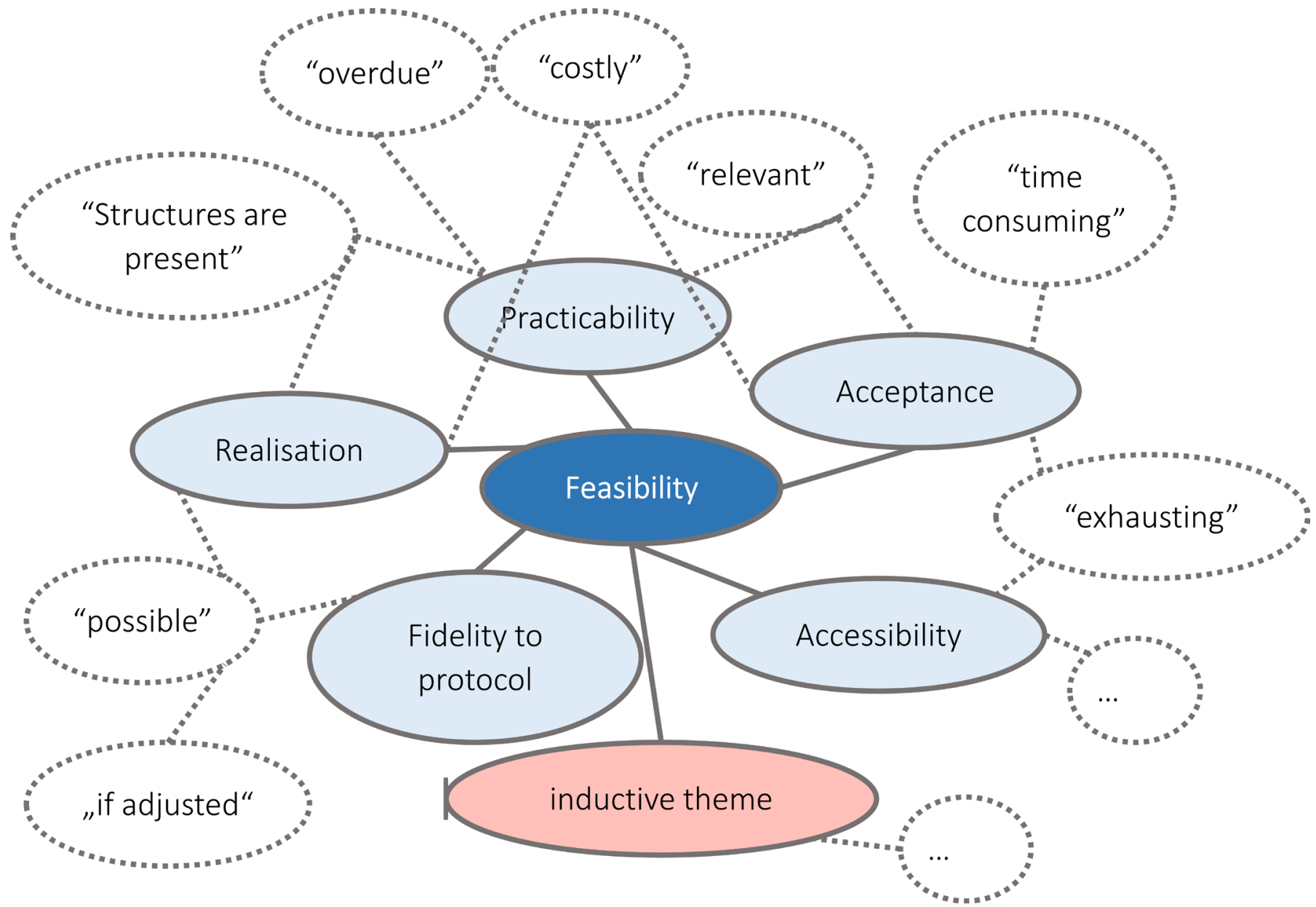

Figure 3 Scheme of theme coding of qualitative feasibility interviews. Potential statements of patients and staff are coded into the different organising aspects of the global feasibility theme.

beforehand and discussed regularly. Nevertheless, we are open to the possibility of inductive theme generation, if data suggests. The results will be reported using consolidated criteria for reporting qualitative research. ${ }^{71}$

\section{Patient and public involvement}

Patients and public were and will not be directly involved in the research study design. However, within the qualitative analysis, we will assess the patient's opinion of the PeriAge intervention, and about burden and time required to take part in this study. One research question is dedicated to obtain and integrate the patient's opinion into the results and eventually into the decision whether to continue and incorporate the programme in routine care. It is not planned to involve patients in the dissemination of the results. If the intervention shows to be feasible and brings added value into the healthcare of geriatric patients, it will be maintained and expanded to all wards and all surgical geriatric patients in the University Medical Centre Hamburg-Eppendorf.

\section{Software}

Microsoft Access will be used for data collection, storage and preparation. For most quantitative data analyses, it is anticipated to use the software $\mathrm{R}^{72}$ and IBM SPSS
Statistics. ${ }^{73}$ Lastly, the software MAXQDA ${ }^{74}$ will be used for qualitative data analyses.

\section{ETHICS AND DISSEMINATION \\ Ethical and safety considerations}

The study will be carried out according to the Helsinki Declaration of the World Medical Association. The principles of good scientific practice will be followed. Study participation is voluntary and may be withdrawn at any moment. Written informed consent will be obtained prior to participation. Patients will be fully educated about the aims and procedure of the study, data collection and the use of collected data. The rejection of participation has no negative consequences for patients and their care. No foreseeable risk at any moment results from the participation in this study. No compassionate use will be carried out. All intervention components are non-invasive expect for the preoperative iron infusion if required according to the Patient Blood Management protocol. However, this is no experimental therapy method but an established and evidence-based measure, which is executed according to existing guidelines and approved by the local ethical review committee. Preserving principles of data sensitivity, 
data protection and confidentiality requirements will be met. Significant deviations from the protocol, concerning recruitment, inclusion criteria, intervention or statistical data analysis will be justified and discussed. Modifications and amendments will be listed in the appendices of the main publication. Standard Protocol Items: Recommendations for Interventional Trials reporting guidelines have been used to write protocol. ${ }^{75}$

\section{Dissemination plan}

The results of the project will be published in scientific journals. In order to assure high accessibility, we aim to publish our work in open access journals, conditions permitting. Furthermore, the results will be presented at relevant national and international conferences. Additionally, a data basis shall be created that will help to inform clinical practice guidelines that enable and improve perioperative care and surgical outcomes of geriatric patients, respectively.

\section{Data deposition}

The collected data will be deposed on a protected server of the University Medical Centre Hamburg-Eppendorf, with strongly regulated access even for study personnel. Due to substantial obstacles to de-identification (relatively small sample, routine care, a large amount of qualitative data, etc), individual participant data will not be shared publicly. Researchers who submit a methodologically sound proposal to the principal investigator that is approved by the responsible review committee will be allowed to use data.

Acknowledgements G. Ketels and M. Lemke, both from the Department of Physiotherapy, UKE, for the development and supervision of the prehabilitationtraining program. R. Schulz, J. Jannsen and C. Raschke: for performing as models in the prehabilitation brochure. C. Langebrake from the UKE Pharmacy for the supervision of the PeriAge medication reconciliation. S. Bargel and G. Ohm from the UKE Developing department, for their support in submitting the grant proposal.

Contributors $\mathrm{CO}, \mathrm{MH}$, RK and LK conceptualised the study, wrote the grant proposal and obtained funding. $L L, C 0, A M$ and $L K$ designed the details of the study, with substantial contributions from MH and RK. RK is the responsible primary investigator of the project. LL and CO prepared the first draft of the manuscript. LP substantially contributed to implementing the individual interventions and the recruitment of patients. AEG and CZ, heads of the UKE Anaesthesiology Department, supported and enabled the realisation of the study with their overall supervision. All authors contributed to critically revising the manuscript for important intellectual content, gave final approval of the version to be published and agree to be accountable for the work as guarantors. The corresponding author attests that all listed authors meet authorship criteria and that no others meeting the criteria have been omitted.

Funding This work is funded by the Innovation Fund of the German Federal Joint Committee (Gemeinsamer Bundesausschuss), with the grant number 01VSF16057 (contact information: phone +49 3027 58380, email: info@g-ba.de). The German Federal Joint Committee reviewed and approved the study design during the grant application process. It had no role in the conduct of the study and the publication process.

Competing interests None declared.

Patient consent for publication Not required.

Ethics approval Ethics Committee of the Medical Association Hamburg, Germany (study ID: PV5596).

Provenance and peer review Not commissioned; externally peer reviewed.

Open access This is an open access article distributed in accordance with the Creative Commons Attribution Non Commercial (CC BY-NC 4.0) license, which permits others to distribute, remix, adapt, build upon this work non-commercially, and license their derivative works on different terms, provided the original work is properly cited, appropriate credit is given, any changes made indicated, and the use is non-commercial. See: http://creativecommons.org/licenses/by-nc/4.0/.

\section{REFERENCES}

1 Fallpauschalenbezogene Krankenhausstatistik (DRG-Statistik), Diagnosen und Prozeduren Der vollstationären Patientinnen und Patienten in Krankenhäusern, Fachserie 12 Reihe 6.4 Statistisches Bundesamt (Destatis); 2017. https://www.destatis.de/DE/ Publikationen/Thematisch/Gesundheit/Krankenhaeuser/Fallpauschal enKrankenhaus.html [17.08.2018]

2 Olotu-Steffen C, Gurlit S, Kiefmann R. Präoperative Vorbereitung und evaluation: Der ältere patient. Anästhesiol Intensivmed Notfallmed Schmerzther 2017;52:342-55.

3 Baquero GA, Rich MW. Perioperative care in older adults. J Geriatr Cardiol 2015;12:465-9.

4 Deiner S, Westlake B, Dutton RP. Patterns of surgical care and complications in elderly adults. J Am Geriatr Soc 2014;62:829-35.10.1111/jgs.12794

5 Lienhart A, Auroy Y, Péquignot F, et al. Survey of Anesthesia-related mortality in France. Anesthesiology 2006;105:1087-97.

6 Sepehri A, Beggs T, Hassan A, et al. The impact of frailty on outcomes after cardiac surgery: a systematic review. J Thorac Cardiovasc Surg 2014;148:3110-7.

7 Patel N, Minhas JS, Chung EML. Risk factors associated with cognitive decline after cardiac surgery: a systematic review. Cardiovasc Psychiatry Neurol 2015;2015:1-12.

8 Rundshagen I. Postoperative cognitive dysfunction. Dtsch Arztebl Int 2014;111:119-25.

$9 \mathrm{Kim}$ S, Brooks AK, Groban L. Preoperative assessment of the older surgical patient: honing in on geriatric syndromes. Clin Interv Aging 2015;10:13-27.

10 Carlisle JB. Pre-Operative co-morbidity and postoperative survival in the elderly: beyond one lunar orbit. Anaesthesia 2014;69:17-25.

11 Newman MF, Kirchner JL, Phillips-Bute B, et al. Longitudinal assessment of neurocognitive function after coronary-artery bypass surgery. N Engl J Med Overseas Ed 2001;344:395-402.

12 Scandrett KG, Zuckerbraun BS, Peitzman AB. Operative risk stratification in the older adult. Surg Clin North Am 2015;95:149-72.

13 Fried LP, Tangen CM, Walston J, et al. Frailty in older adults: evidence for a phenotype. The Journals of Gerontology Series A: Biological Sciences and Medical Sciences 2001;56:M146-57.

14 Beggs T, Sepehri A, Szwajcer A, et al. Frailty and perioperative outcomes: a narrative review. Can J Anesth/J Can Anesth 2015;62:143-57.

15 Aunan JR, Watson MM, Hagland HR, et al. Molecular and biological hallmarks of ageing. Br J Surg 2016;103:e29-46.

16 Baron R, Binder A, Biniek R, et al. Evidence and consensus based guideline for the management of delirium, analgesia, and sedation in intensive care medicine. Revision 2015 (DAS-Guideline 2015) - short version. Ger Med Sci 2015;13.

17 Bauer JM, Volkert D, Wirth R, et al. Diagnostik Der Mangelernährung des älteren Menschen. Dtsch med Wochenschr 2006;131:223-7.

18 Weimann A, Braga M, Harsanyi L, et al. ESPEN guidelines on enteral nutrition: surgery including organ transplantation. Clin Nutr 2006;25:224-44.

19 Jack S, West M, Grocott MPW. Perioperative exercise training in elderly subjects. Best Pract Res Clin Anaesthesiol 2011;25:461-72.10.1016/..bpa.2011.07.003

20 Deutz NEP, Bauer JM, Barazzoni R, et al. Protein intake and exercise for optimal muscle function with aging: recommendations from the ESPEN expert group. Clinical Nutrition 2014;33:929-36.

21 Mohanty S, Rosenthal RA, Russell MM, et al. Optimal perioperative management of the geriatric patient: a best practices guideline from the American College of surgeons NSQIP and the American geriatrics Society. J Am Coll Surg 2016;222:930-47.

22 By the American Geriatrics Society 2015 Beers Criteria Update Expert Panel. American geriatrics Society 2015 updated beers criteria for potentially inappropriate medication use in older adults. $J$ Am Geriatr Soc 2015;63:2227-46.

23 Holt S, Schmiedl S, Thürmann PA. Potentially inappropriate medications in the elderly: the PRISCUS list. Dtsch Arztebl Int 2010;107:543-51.

24 Smith I, Kranke P, Murat I, et al. Perioperative fasting in adults and children: guidelines from the European Society of Anaesthesiology. Eur J Anaesthesiol 2011;28:556-69.

25 Deutsche Gesellschaft für Anästhesiologie und Intensivmedizin e.V., Berufsverband Deutscher Anästhesisten e.V.. „Entschließungen Empfehlungen - Vereinbarungen “ Ein Beitrag zur Qualitätssicherung 
in der Anästhesiologie. 5. DGAI und BDA, 2011. ISBN: 978-3932653-36-0.

26 Ali Abdelhamid Y, Chapman MJ, Deane AM. Peri-Operative nutrition. Anaesthesia 2016;71 Suppl 1:9-18.

27 Broadbent E, Petrie KJ, Alley PG, et al. Psychological stress impairs early wound repair following surgery. Psychosom Med 2003;65:865-9.

28 Wozniak SE, Coleman J, Katlic MR. Optimal preoperative evaluation and perioperative care of the geriatric patient. Anesthesiol Clin 2015;33:481-9.

29 Inouye SK, Bogardus ST, Baker DI, et al. The hospital elder life program: a model of care to prevent cognitive and functional decline in older hospitalized patients. Hospital elder life program. J Am Geriatr Soc 2000;48:1697-706.

30 Muñoz M, Acheson AG, Auerbach M, et al. International consensus statement on the peri-operative management of anaemia and iron deficiency. Anaesthesia 2017;72:233-47.

31 Mehra T, Seifert B, Bravo-Reiter S, et al. Implementation of a patient blood management monitoring and feedback program significantly reduces transfusions and costs. Transfusion 2015;55:2807-15

32 Griffiths R, Beech F, Brown A, et al. Peri-Operative care of the elderly 2014: association of anaesthetists of great britain and ireland. Anaesthesia 2014;69:81-98.

33 Gombotz H, Zacharowski K, Spahn DR, et al. Patient blood management - Individuelles Behandlungskonzept zur Reduktion und Vermeidung von Anämie. 1. Stuttgart, New York: Georg Thieme Verlag, 2013. ISBN: 9783131706218 . https://www.thieme-connect. de/products/ebooks/book/10.1055/b-002-59191

34 Radtke FM, Franck M, Lendner J, et al. Monitoring depth of anaesthesia in a randomized trial decreases the rate of postoperative delirium but not postoperative cognitive dysfunction. $\mathrm{Br} J$ Anaesth 2013;110:i98-105

35 Vaurio LE, Sands LP, Wang Y, et al. Postoperative delirium: the importance of pain and pain management. Anesthesia \& Analgesia 2006;102:1267-73.

36 Rundshagen I. Anästhesiologische Strategien bei Hochbetagten. Anästhesie und Intensivmedizin 2015;56:534-45.

37 Varadhan KK, Neal KR, Dejong CHC, et al. The enhanced recovery after surgery (ERAS) pathway for patients undergoing major elective open colorectal surgery: a meta-analysis of randomized controlled trials. Clinical Nutrition 2010;29:434-40.

38 Santa Mina D, Clarke H, Ritvo P, et al. Effect of total-body prehabilitation on postoperative outcomes: a systematic review and meta-analysis. Physiotherapy 2014;100:196-207.

39 Rubin FH, Williams JT, Lescisin DA, et al. Replicating the hospital elder life program in a community hospital and demonstrating effectiveness using quality improvement methodology. J Am Geriatr Soc 2006;54:969-74.

40 Damschroder LJ, Aron DC, Keith RE, et al. Fostering implementation of health services research findings into practice: a consolidated framework for advancing implementation science. Implementation Sci 2009;4.

41 Partridge JSL, Harari D, Martin FC, et al. The impact of pre-operative comprehensive geriatric assessment on postoperative outcomes in older patients undergoing scheduled surgery: a systematic review. Anaesthesia 2014;69:8-16.

42 Lawton MP, Brody EM. Assessment of older people: selfmaintaining and instrumental activities of daily living. Gerontologist 1969;9:179-86.

43 Moore GF, Audrey S, Barker M, et al. Process evaluation of complex interventions: medical Research Council guidance. $B M J$ 2015;350:h1258.

44 Melnyk M, Casey RG, Black P, et al. Enhanced recovery after surgery (ERAS) protocols: time to change practice? Can Urol Assoc $J$ 2011;5:342-8.

45 Kalbe E, Kessler J, Calabrese P, et al. DemTect: a new, sensitive cognitive screening test to support the diagnosis of mild cognitive impairment and early dementia. Int $J$ Geriatr Psychiatry 2004;19:136-43.

46 Zimmermann P, Fimm B. A test battery for attentional performance. In: Applied neuropsychology of attention - Theory, Diagnosis and Rehabilitation. 1. Psychology Press, 2004: 124-65. ISBN: 9780203307014. https://www.taylorfrancis.com/books/e/ 9780203307014

47 Reitan RM, Wolfson D. Conventional intelligence measurements and neuropsychological concepts of adaptive abilities. J Clin Psychol 1992:48:521-9.

48 Ware J, Kosinski M, Keller SD. A 12-Item short-form health survey: construction of scales and preliminary tests of reliability and validity. Med Care 1996;34:220-33.
49 Millor N, Lecumberri P, Gomez M, et al. Gait velocity and chair SitStand-Sit performance improves current Frailty-Status identification. IEEE Trans Neural Syst Rehabil Eng 2017;25:2018-25.

50 Phillips P, strength G. Grip strength, mental performance and nutritional status as indicators of mortality risk among female geriatric patients. Age Ageing 1986;15:53-6.

51 Bohannon RW. Sit-to-stand test for measuring performance of lower extremity muscles. Percept Mot Skills 1995;80:163-6.

52 Aldecoa C, Bettelli G, Bilotta F, et al. European Society of Anaesthesiology evidence-based and consensus-based guideline on postoperative delirium. Eur J Anaesthesiol 2017;34:192-214.

53 Smith MD, McCall J, Plank L, et al. Preoperative carbohydrate treatment for enhancing recovery after elective surgery. Cochrane Database Syst Rev 2014;88.

54 Kaška M, Grosmanová Tat'ána, Havel E, et al. The impact and safety of preoperative oral or intravenous carbohydrate administration versus fasting in colorectal surgery - a randomized controlled trial. Wien Klin Wochenschr 2010;122:23-30.

55 Viechtbauer W, Smits L, Kotz D, et al. A simple formula for the calculation of sample size in pilot studies. J Clin Epidemiol 2015;68:1375-9.

56 Billingham SAM, Whitehead AL, Julious SA. An audit of sample sizes for pilot and feasibility trials being undertaken in the United Kingdom registered in the United Kingdom clinical research network database. BMC Med Res Methodol 2013;13:104.

57 Story DA. Postoperative complications in elderly patients and their significance for long-term prognosis. Curr Opin Anaesthesiol 2008;21:375-9.

58 McNicol L, Story DA, Leslie K, et al. Postoperative complications and mortality in older patients having non-cardiac surgery at three Melbourne teaching hospitals. Med J Aust 2007;186:447-52.

59 Ommundsen N, Wyller TB, Nesbakken A, et al. Preoperative geriatric assessment and tailored interventions in frail older patients with colorectal cancer: a randomized controlled trial. Colorectal Dis 2018;20:16-25

60 Deschodt M, Braes T, Broos P, et al. Effect of an inpatient geriatric consultation team on functional outcome, mortality, institutionalization, and readmission rate in older adults with hip fracture: a controlled trial. J Am Geriatr Soc 2011;59:1299-308.

61 Wagner AK, Soumerai SB, Zhang F, et al. Segmented regression analysis of interrupted time series studies in medication use research. J Clin Pharm Ther 2002;27:299-309.

62 Gebski V, Ellingson K, Edwards J, et al. Modelling interrupted time series to evaluate prevention and control of infection in healthcare. Epidemiol Infect 2012;140:2131-41.

63 Kontopantelis E, Doran T, Springate DA, et al. Regression based quasi-experimental approach when randomisation is not an option: interrupted time series analysis. BMJ 2015;350:h2750.10.1136/bmj. h2750

64 ROSENBAUM PR, RUBIN DB. The central role of the propensity score in observational studies for causal effects. Biometrika 1983;70:41-55.

65 Craig P, Dieppe P, Macintyre S, et al. Developing and evaluating complex interventions: the new medical Research Council guidance. BMJ 2008;337.

66 Glasgow RE, McKay HG, Piette JD, et al. The RE-AIM framework for evaluating interventions: what can it tell us about approaches to chronic illness management? Patient Educ Couns 2001;44:119-27.

67 Proctor E, Silmere H, Raghavan R, et al. Outcomes for implementation research: conceptual distinctions, measurement challenges, and research agenda. Adm Policy Ment Health 2011;38:65-76.

68 Marshall MN. Sampling for qualitative research. Fam Pract 1996;13:522-6.

69 Braun V, Clarke V. Using thematic analysis in psychology. Qual Res Psychol 2006;3:77-101.

70 Gale NK, Heath G, Cameron E, et al. Using the framework method for the analysis of qualitative data in multi-disciplinary health research. BMC Med Res Methodol 2013;13:117.

71 Tong A, Sainsbury P, Craig J. Consolidated criteria for reporting qualitative research (COREQ): a 32-item checklist for interviews and focus groups. Int J Qual Health Care 2007;19:349-57.

72 R Core Team. R: a language and environment for statistical computing. $\mathrm{R}$ foundation for statistical computing. Vienna, Austria. URL. Available: https://www.blopig.com/blog/2013/07/citing-rpackages-in-your-thesispaperassignmentsthesispaperassignments/ [Accessed 12.11.2018].

73 IBM Corp. IBM SPSS Statistics for Windows, Version 23.0. Armonk NY: IBM Corp, 2015.

74 VERBI S. MAXQDA 2018 [computer software]. Berlin, Germany: VERBI Software. Available: https://www.maxqda.com 
75 Chan A-W, Tetzlaff JM, Gøtzsche PC, et al. Spirit 2013 explanation and elaboration: guidance for protocols of clinical trials. $B M J$ 2013;346:e7586.

76 Zemke J, Situation EderS. Erfassung Der Sozialen situation (SOS) nACh Nikolaus. GGP 2018;02:260-2.

77 Podsiadlo D, Richardson S. The timed "Up \& Go": a test of basic functional mobility for frail elderly persons. J Am Geriatr Soc 1991;39:142-8

78 Dapp U, Anders J, von Renteln-Kruse W, et al. The longitudinal urban cohort ageing study (LUCAS): study protocol and participation in the first decade. BMC Geriatr 2012;12:35.

79 Skates JJ, Anthony PS. Identifying Geriatric Malnutrition in Nursing Practice: The Mini Nutritional Assessment (MNA®) - An EvidenceBased Screening Tool. J Gerontol Nurs 2012;38:18-27.
80 Ely EW, Margolin R, Francis J, et al. Evaluation of delirium in critically ill patients: validation of the confusion assessment method for the intensive care unit (CAM-ICU). Crit Care Med 2001;29:1370-9.

81 Morfeld M, Bullinger M, Kirchberger J. Sf-36 Fragebogen zum Gesundheitszustand. Deutsche version des short Form-36 health survey. . Hogrefe Verlag, Göttingen, 2011: 2. 1-222.

82 Yesavage JA, Brink TL, Rose TL, et al. Development and validation of a geriatric depression screening scale: a preliminary report. $J$ Psychiatr Res 1982;17:37-49.

83 Spitzer RL, Kroenke K, Williams JBW, et al. A brief measure for assessing generalized anxiety disorder. Arch Intern Med 2006;166:1092-7.10.1001/archinte.166.10.1092

84 Le Manach Y, Collins G, Rodseth R, et al. Preoperative score to predict postoperative mortality (POSPOM): derivation and validation. Anesthesiology 2016;124:570-9. 